TITLE:

\title{
Characterization of hydroxy fatty acid dehydrogenase involved in polyunsaturated fatty acid saturation metabolism in Lactobacillus plantarum AKU 1009a
}

\section{AUTHOR(S):}

Takeuchi, Michiki; Kishino, Shigenobu; Park, SiBum; Kitamura, Nahoko; Ogawa, Jun

\section{CITATION:}

Takeuchi, Michiki ...[et al]. Characterization of hydroxy fatty acid dehydrogenase involved in polyunsaturated fatty acid saturation metabolism in Lactobacillus plantarum AKU 1009a. Journal of Molecular Catalysis B: Enzymatic 2015, 117: 7-12

\section{ISSUE DATE:}

2015-04-06

\section{URL:}

http://hdl.handle.net/2433/198740

\section{RIGHT:}

(c) 2015 Elsevier B.V. Licensed under the Creative Commons Attribution-NonCommercial-NoDerivatives 4.0 International http://creativecommons.org/licenses/by-nc-nd/4.0/. NOTICE: this is the author's version of a work that was accepted for publication in <Journal of Molecular Catalysis B: Enzymatic>. Changes resulting from the publishing process, such as peer review, editing, corrections, structural formatting, and other quality control mechanisms may not be reflected in this document. Changes may have been made to this work since it was submitted for publication. A definitive version was subsequently published in [Journal of Molecular Catalysis B: Enzymatic, Volume 117, Pages 7-12] doi:10.1016/j.molcatb.2015.03.020.; 許諾条件により本文ファイルは2017-04-06に公開.; This is not the published version. Please cite only the published version.; この論文は出版社版でありません。引用の際には出版社版をご確認ご 利用ください。 
1 Characterization of hydroxy fatty acid dehydrogenase involved in polyunsaturated fatty

2 acid saturation metabolism in Lactobacillus plantarum AKU 1009a

3 Running title: Hydroxy fatty acid dehydrogenase from L. plantarum

4 Michiki Takeuchi ${ }^{1}$, Shigenobu Kishino ${ }^{1,2, *}$, Si-Bum Park $^{2}$, Nahoko Kitamura ${ }^{1}$, Jun

$5 \quad$ Ogawa $^{1,3, *, \dagger}$

$6 \quad{ }^{1}$ Division of Applied Life Sciences, Graduate School of Agriculture, Kyoto University,

7 Kitashirakawa-oiwakecho, Sakyo-ku, Kyoto, 606-8502 Japan,

8 2 Laboratory of Industrial Microbiology, Graduate School of Agriculture, Kyoto

9 University, Kitashirakawa-oiwakecho, Sakyo-ku, Kyoto, 606-8502 Japan

$10{ }^{3}$ Research Unit for Physiological Chemistry, Kyoto University, Kyoto, Japan

$11 *$ These corresponding authors contributed equally to this work.

$13{ }^{\dagger}$ Corresponding author. Tel.: +81 75753 6115, Fax: +81 757536113.

14 E-mail address: ogawa@kais.kyoto-u.ac.jp (J. Ogawa). 


\section{ABSTRACT}

Hydroxy fatty acid dehydrogenase, which is involved in polyunsaturated fatty acid saturation metabolism in Lactobacillus plantarum AKU 1009a, was cloned, expressed, purified, and characterized. The enzyme preferentially catalyzed NADH-dependent hydrogenation of oxo fatty acids over $\mathrm{NAD}^{+}$-dependent dehydrogenation of hydroxy fatty acids. In the dehydrogenation reaction, fatty acids with an internal hydroxy group such as 10-hydroxy-cis-12-octadecenoic acid, 12-hydroxy-cis-9-octadecenoic acid, and 13-hydroxy-cis-9-octadecenoic acid served as better substrates than those with $\alpha$ - or $\beta$-hydroxy groups such as 3-hydroxyoctadecanoic acid or 2-hydroxyeicosanoic acid. The apparent $K_{m}$ value for 10-hydroxy-cis-12-octadecenoic acid (HYA) was estimated to be $38 \mu \mathrm{M}$ with a $k_{\text {cat }}$ of $7.6 \cdot 10^{-3} \mathrm{~s}^{-1}$. The apparent $K_{m}$ value for 10-oxo-cis-12-octadecenoic acid (KetoA) was estimated to be $1.8 \mu \mathrm{M}$ with a $k_{\text {cat }}$ of $5.7 \cdot 10^{-1} \mathrm{~s}^{-1}$. In the hydrogenation reaction of KetoA, both $(R)$ - and $(S)$ - HYA were generated, indicating that the enzyme has low stereoselectivity. This is the first report of a dehydrogenase with a preference for fatty acids with an internal hydroxy group.

Keywords: Lactic acid bacteria; Hydroxy fatty acid; Oxo fatty acid; short-chain dehydrogenase/reductase 


\section{Introduction}

Functional lipids have attracted attention both nutritionally and pharmaceutically.

Conjugated linoleic acid (CLA) is a representative functional fatty acid, which has beneficial effects such as decreasing body fat content [1] and preventing tumorigenesis [2,3] and arteriosclerosis [4]. Oxo fatty acids as well as CLA have also been proven to have novel physiological functions. For example, it has recently been reported that 13-oxo-9,11-octadecadienoic acid in tomato juice acts as a potent peroxisome proliferator activated receptor $\alpha(\mathrm{PPAR} \alpha)$ agonist and improves dyslipidemia and hepatic steatosis induced by obesity [5].

In our previous study, we revealed polyunsaturated fatty acid saturation metabolism in Lactobacillus plantarum AKU 1009a [6], which is a strain with a potential to produce CLA from linoleic acid [7-10]. The novel saturation metabolism consisted of four enzymes: CLA-HY (hydratase/dehydratase) [6,11,12], CLA-DH (dehydrogenase), CLA-DC (isomerase), and CLA-ER (enone reductase) [6,12]. This saturation metabolism included some oxo fatty acids, such as 10-oxo-cis-12-octadecenoic acid

50 (KetoA), 10-oxooctadecanoic acid, and 10-oxo-trans-11-octadecenoic acid, as intermediates. These oxo fatty acids are expected to have new physiological activities. 
53 hydroxy fatty acids, e.g., dehydrogenation of HYA to KetoA.

54 In this study, we describe the enzymatic and physiochemical characteristics of

55 CLA-DH, which is involved in the saturation metabolism and catalyzes the dehydrogenation of hydroxy fatty acids and the hydrogenation of oxo fatty acids.

\section{Materials and methods}

\subsection{Chemicals}

60

HYA, 10-hydroxyoctadecanoic acid, 10-hydroxy-trans-11-octadecenoic acid,

\section{1 (S)-10-hydroxy-cis-12,cis-15-octadecadienoic}

acid,

(S)-10-hydroxy-cis-6,cis-12-octadecadienoic acid, and 13-hydroxy-cis-9-octadecenoic

acid were prepared as previously described $[6,11,13,14]$. Oxo fatty acids (KetoA,

acid,

10-oxo-cis-12,cis-15-octadecadienoic acid, 10-oxo-cis-6,cis-12-octadecadienoic acid,

66 12-oxo-cis-9-octadecenoic acid, and 13-oxo-cis-9-octadecenoic acid) were prepared

67 from hydroxy fatty acids (HYA, 10-hydroxyoctadecanoic acid,

68 10-hydroxy-trans-11-octadecenoic acid, (S)-10-hydroxy-cis-12,cis-15-octadecadienoic

69 acid, (S)-10-hydroxy-cis-6,cis-12-octadecadienoic acid, ricinoleic acid, and

70 13-hydroxy-cis-9-octadecenoic acid) by Jones oxidation, which is oxidation of the 
hydroxy group with $\mathrm{CrO}_{3}[15]$. (R)-HYA was purified from racemic HYA using HPLC equipped with a chiral column in the same method as "Enantiomeric purity analysis of hydroxy fatty acids” shown in below. Fatty acid-free $(<0.02 \%)$ bovine serum albumin (BSA) was purchased from Sigma (St. Louis, USA). All other chemicals were of analytical grade and were commercially obtained.

\subsection{Preparation of CLA-DH}

Escherichia coli Rosetta2/pCLA-DH [12] cells were cultured in $1.5 \mathrm{~L}$ of Luria-Bertani (LB) medium at $37^{\circ} \mathrm{C}$ for $2 \mathrm{~h}$ with simultaneous shaking at $100 \mathrm{rpm}$, and then isopropyl- $\beta$-thiogalactopyranoside (IPTG) was added to a final concentration of 1.0 $\mathrm{mM}$. After adding IPTG, the transformed cells were cultivated at $20^{\circ} \mathrm{C}$ for $8 \mathrm{~h}$ with simultaneous shaking at $100 \mathrm{rpm}$. After cultivation, the transformed cells (8 g) were harvested, suspended in a standard buffer $(16 \mathrm{~mL})$, and treated with an ultrasonic oscillator (5 min, 4 times, Insinator 201 M; Kubota, Japan). The standard buffer contained $1 \mathrm{mM}$ DTT and 10\% (v/v) ethylene glycol in $20 \mathrm{mM}$ potassium phosphate buffer (KPB) (pH 6.5). The cell debris was removed by centrifuging at $1,700 \mathrm{~g}$ for 10 min. The resulting supernatant solutions were used as cell-free extracts. The cell-free extracts were fractioned ultracentrifugation at $100,000 \mathrm{~g}$ for $60 \mathrm{~min}$ and the supernatant 
89 was obtained. CLA-DH was purified from this supernatant using a fast protein liquid

90 chromatography (FPLC) system (GE Healthcare) equilibrated with the standard buffer.

91 The supernatant was applied to a HiLoad 26/60 Superdex 200 prep-grade column (GE

92 Healthcare) that had already been equilibrated with standard buffer and eluted.

93 CLA-DH was further purified using a Mono Q 10/100 GL column (GE Healthcare), a

94 Superdex 200 10/300 GL column (GE Healthcare), and a Phenyl Superose HR 10/10

95 (Pharmacia). The purified CLA-DH was dialyzed with the standard buffer including $50 \%(\mathrm{v} / \mathrm{v})$ glycerol and stored at $-20^{\circ} \mathrm{C}$ until further use.

97

\subsection{Determination of the molecular mass of CLA-DH}

In order to determine the native molecular mass of CLA-DH, the enzyme solution

was subjected to high performance gel-permeation chromatography on a G-3000SW column $(0.75 \times 60 \mathrm{~cm}$, Tosoh, Tokyo, Japan $)$ at room temperature. It was eluted with $100 \mathrm{mM} \mathrm{KPB}\left(\mathrm{pH}\right.$ 6.5) containing $100 \mathrm{mM} \mathrm{Na}_{2} \mathrm{SO}_{4}$ at a flow rate of $0.5 \mathrm{~mL} / \mathrm{min}$. The absorbance of the effluent was monitored at $280 \mathrm{~nm}$. The molecular mass of the enzymes was determined from their mobility relative to those of standard proteins. 
107 All operations were performed in an anaerobic chamber. The standard reaction

108 conditions were as described. The reactions were performed in test tubes $(16.5 \times 125$

$109 \mathrm{~mm}$ ) that contained $1 \mathrm{~mL}$ of reaction mixture (20 mM sodium succinate buffer, $\mathrm{pH} 4.5)$

110 with $0.1 \%(\mathrm{w} / \mathrm{v})$ HYA or KetoA complexed with BSA $[0.02 \%(\mathrm{w} / \mathrm{v})]$ as the substrate, 5

$111 \mathrm{mM} \mathrm{NAD}^{+}$or NADH and $42 \mu \mathrm{g}(=0.04 \mathrm{U} / \mathrm{ml})$ purified CLA-DH. One unit was defined

112 as the amount of enzyme that catalyzes the conversion of $1 \mu \mathrm{mol}$ of HYA per minute.

113 The reactions were performed under anaerobic conditions in a sealed chamber with an

$114 \mathrm{O}_{2}$-absorbent (Anaeropack “Kenki,” Mitsubishi Gas Chemical Co., Ltd., Tokyo, Japan)

115 and gently shaken (120 strokes $/ \mathrm{min})$ at $37^{\circ} \mathrm{C}$ for $15 \mathrm{~min}$. All experiments were

116 performed in triplicate. Reactions were performed under the standard reaction

117 conditions with some modifications, as described below. The optimal reaction

118 temperature was determined by incubating $1 \mathrm{~mL}$ of the reaction mixture $(20 \mathrm{mM}$

119 sodium succinate buffer, $\mathrm{pH}$ 4.5) at various temperatures for 15 min under anaerobic

120 conditions. The optimal reaction $\mathrm{pH}$ was determined at $37^{\circ} \mathrm{C}$ using $1 \mathrm{~mL}$ of $20 \mathrm{mM}$

121 sodium citrate buffer ( $\mathrm{pH} 3.0-4.0$ ) or $20 \mathrm{mM}$ sodium succinate buffer ( $\mathrm{pH} 4.0-5.5$ ).

122 Thermal stability was determined by measuring the enzyme activity after incubating 1

$123 \mathrm{~mL}$ of reaction mixture containing $20 \mathrm{mM}$ sodium succinate buffer ( $\mathrm{pH} 4.5)$ at various

124 temperatures for 15 min under anaerobic conditions. The $\mathrm{pH}$ stability was determined 
125 by measuring enzyme activity after incubating at $37^{\circ} \mathrm{C}$ for $10 \mathrm{~min}$ in the following

126 buffers under anaerobic conditions: sodium citrate buffer (50 mM; pH 3.0-4.0), sodium

127 succinate buffer (50 mM; pH 4.0-6.0), KPB (50 mM; pH 5.5-8.0), and Tris-HCl buffer 128 (50 mM; pH 7.0-9.0).

129

\subsection{Kinetic analysis}

131

All procedures were performed in an anaerobic chamber. Reactions were

performed under standard reaction conditions with modified substrate and enzyme

concentrations. The kinetics of HYA dehydrogenation were studied using 30-1000 $\mu \mathrm{M}$

134 HYA complexed with $0.02 \%(\mathrm{w} / \mathrm{v})$ BSA as the substrate, $7 \mu \mathrm{g} / \mathrm{mL}$ CLA-DH, and a

135 reaction time of 15 min. The kinetics of KetoA dehydrogenation were studied using

$1361-20 \mu \mathrm{M}$ KetoA complexed with $0.02 \%(\mathrm{w} / \mathrm{v})$ BSA as the substrate, $0.35 \mu \mathrm{g} / \mathrm{mL}$

137 CLA-DH, and a reaction time of $5 \mathrm{~min}$. The kinetic parameters were calculated by using

138 the experimental data with the Michaelis-Menten equation using KaleidaGraph 4.0

139 (Synergy Software Inc., PA, USA).

140

\subsection{Lipid analysis}

142 Before lipid extraction, $n$-heptadecanoic acid was added to the reaction mixture as an 
143 internal standard. Lipids were extracted from $1 \mathrm{~mL}$ of the reaction mixture using $5 \mathrm{~mL}$

144 of chloroform/methanol/1.5\% (w/v) $\mathrm{KCl}$ in $\mathrm{H}_{2} \mathrm{O}$ (2:2:1, by volume) according to the

145 procedure of Bligh-Dyer, and then concentrated by evaporation under reduced pressure

146 [16]. The resulting lipids were dissolved in $5 \mathrm{~mL}$ of benzene/methanol (3:2, by volume)

147 and methylated with $300 \mu \mathrm{L}$ of $1 \%$ trimethylsilyldiazomethane (in hexane) at $28^{\circ} \mathrm{C}$ for

14830 min. After methyl esterification, the resulting fatty acid methyl esters were

149 concentrated by evaporation under reduced pressure. The resulting fatty acid methyl

150 esters were analyzed by gas-liquid chromatography (GC) using a Shimadzu (Kyoto,

151 Japan) GC-1700 gas chromatograph equipped with a flame ionization detector, a split

152 injection system, and a capillary column (SPB-1, $30 \mathrm{~m} \times 0.25 \mathrm{~mm}$ I.D., SUPELCO, PA,

153 USA). The initial column temperature $180^{\circ} \mathrm{C}$ (for $30 \mathrm{~min}$ ) was subsequently increased

154 to $210^{\circ} \mathrm{C}$ at a rate of $60^{\circ} \mathrm{C} / \mathrm{min}$, and then maintained at $210^{\circ} \mathrm{C}$ for $29.5 \mathrm{~min}$. The injector

155 and detector were operated at $250^{\circ} \mathrm{C}$. Helium was used as a carrier gas at a flow rate of

$1561.4 \mathrm{~mL} / \mathrm{min}$. The fatty acid peaks were identified by comparing the retention times to

157 those of known standards.

\subsection{Enantiomeric purity analysis of hydroxy fatty acids}

160 The enantiomeric purity of HYA, which was produced from KetoA hydrogenation 
161 with CLA-DH, was analyzed using HPLC (Shimadzu, Kyoto, Japan) using a Shimadzu

162 LC 20A System (Shimadzu) equipped with a chiral column (Chiralpak IA, 4.6 mm I.D.,

163 Daicel, Osaka, Japan) and an Evaporative Light Scattering Detector System (Shimadzu,

164 Kyoto, Japan) as a detector. Acetonitrile/0.2\% formic acid (65:35) was used as a solvent

165 at a flow rate of $1.0 \mathrm{~mL} / \mathrm{min}$. Racemic HYA prepared from KetoA reduction with

$166 \mathrm{NaBH}_{4}[17]$ was used as the standard.

167

168

\section{Results}

\subsection{Purification of CLA-DH}

170

The recombinant CLA-DH without the tag was purified to homogeneity from cell-free extracts of the transformed E. coli through four steps of column chromatography. The purified CLA-DH displayed a single band on an SDS-PAGE gel

(Fig. 1). The observed molecular mass of the subunit was $40 \mathrm{kDa}$, corresponding to a calculated mass of $32 \mathrm{kDa}$ deduced from the amino acid sequence of its gene. The relative native molecular mass was estimated to be $32 \mathrm{kDa}$ by HPLC on a G-3000SW column, indicating that the enzyme consists of the single subunit. The purified CLA-DH was used for further characterization. 


\subsection{Effects of reaction conditions} of $\mathrm{NAD}^{+} / \mathrm{NADH}$ concentration were examined from 0 to $7.5 \mathrm{mM}$ (Fig. 2a). The of $\mathrm{NAD}^{+} / \mathrm{NADH}$. The effects of temperature were also examined. The optimal reaction $\mathrm{pH}$ range from 3.0 to 5.5 with an optimal reaction $\mathrm{pH}$ determined to be $\mathrm{pH} 4.5$ (Fig. 2c).

\subsection{Enzyme stability}

The thermal stability of the purified enzyme was investigated from $18^{\circ} \mathrm{C}$ to $67^{\circ} \mathrm{C}$.

The enzyme was incubated at each temperature for $15 \mathrm{~min}$ at $\mathrm{pH} 4.5$. More than $80 \%$ of the initial activity remained at temperatures up to $28^{\circ} \mathrm{C}$ (Fig. 3a). The $\mathrm{pH}$ stability of the purified enzyme was investigated by incubating the enzyme in different buffers within a $\mathrm{pH}$ range of 3.0 to 9.0 for $10 \mathrm{~min}$ at $37^{\circ} \mathrm{C}$. More than $80 \%$ of the initial activity remained in a pH range from 4.5 to 7.5 (Fig. 3b).

\subsection{Substrate specificity}

In the dehydrogenation reaction, 10-, 12-, or 13-hydroxy C18 fatty acids such as 
197 HYA, (R)-HYA, 10-hydroxyoctadecanoid acid, 10-hydroxy-trans-11-octadecenoic acid,

199 (S)-10-hydroxy-cis-6,cis-12-octadecadienoic acid, (R)-12-hydroxy-cis-9-octadecenoic acid, and 13-hydroxy-cis-9-octadecenoic acid served as good substrates and transformed

201 into corresponding 10-, 12-, or 13-oxo fatty acids. In addition, HYA methyl ester and respectively. In contrast, 2- or 3-hydroxy fatty acids such as 3-hydroxyoctadecanoic acid, 3-hydroxytetradecanoic acid, and 2-hydroxyeicosanoic acid were not dehydrogenated (Table 1). 10-oxo-cis-12,cis-15-octadecadienoic acid, 10-oxo-cis-6,cis-12-octadecadienoic acid, 12-oxo-cis-9-octadecenoic acid, and 13-oxo-cis-9-octadecenoic acid served as good substrates and transformed into corresponding 10-, 12- or 13-hydroxy fatty acids. In addition, KetoA methyl ester and 7-hexadecanone were hydrogenated to HYA methyl ester and 7-hexadecanol, respectively (Table 2). 
The substrate concentration-reaction velocity curves for HYA dehydrogenation and

216 KetoA hydrogenation were used with the Michaelis-Menten equation. The apparent $K_{m}$

217 value for HYA in the dehydrogenation reaction was estimated to be $38 \mu \mathrm{M}$ with a $k_{c a t}$ of

$2187.6 \cdot 10^{-3} \mathrm{sec}^{-1}$. The apparent $K_{m}$ value for KetoA in the hydrogenation reaction was

219 estimated to be $1.8 \mu \mathrm{M}$ with a $k_{\text {cat }}$ of $5.7 \cdot 10^{-1} \mathrm{sec}^{-1}$.

\subsection{Enantiomeric purities of the hydroxy fatty acids produced by CLA-DH}

The enantiomeric purity of HYA produced from KetoA by CLA-DH was analyzed using HPLC with a chiral column. Almost the same amounts of both enantiomers of $(R)$-HYA and (S)-HYA were produced from KetoA, indicating that CLA-DH had low stereoselectivity in oxo fatty acid hydrogenation.

\subsection{Effects of chemicals on the enzyme activity}

The effects of metal ions and inhibitors $(1 \mathrm{mM})$ were investigated in both the

hydration and dehydration reactions. The reactions were strongly inhibited by $\mathrm{Ag}^{+}, \mathrm{Cu}^{2+}$,

$230 \mathrm{Hg}^{2+}, \mathrm{VO}_{3}{ }^{-}, \mathrm{WO}_{4}{ }^{2-}$, and aluminon (data not shown). 2,3,5-Triphenyltetrazolium 


\section{Discussion}

234 We revealed polyunsaturated fatty acid saturation metabolism in L. plantarum AKU

235 1009a and identified CLA-DH. The CLA-DH gene was located together with CLA-DC

236 (fatty acid isomerase) and CLA-ER (fatty acid enone reductase) genes involved in

237 polyunsaturated fatty acid saturation metabolism in L. plantarum AKU 1009a [6]. These

238 results suggested that CLA-DH plays an important role in saturation metabolism.

239 CLA-DH, which belongs to the short-chain dehydrogenase/reductase (SDR) family,

240 showed considerable similarity with other SDRs (Fig. 4). In this paper, we characterized

241 CLA-DH from the aspect of its physiological function to clarify its distinct

242 characteristic properties in the SDR family, especially from the viewpoint of substrate

243 specificity. There are few reports regarding either hydroxy fatty acid dehydrogenation or

244 oxo fatty acid hydrogenation in the SDR family. However, CLA-DH catalyzed the

245 dehydrogenation or hydrogenation of fatty acids which have an internal hydroxy or an

246 oxo group, respectively (Table 1 and 2). Micrococcus luteus WIUJH-20 was reported to

247 convert 10- or 12-hydroxyoctadecanoic acid to the corresponding oxooctadecanoic acid.

248 The amino acid sequence of the enzyme which catalyzes the above oxidation of

249 hydroxy fatty acid in $M$. luteus WIUJH-20 belongs to a secondary alcohol

250 dehydrogenase [18]. The amino acid sequence of the secondary alcohol dehydrogenase 
251 from $M$. luteus did not resemble that of CLA-DH, indicating that the dehydrogenation

252 activity of CLA-DH was characteristic activity among the SDR family.

253 As a characteristic property of CLA-DH, the enzyme showed higher activity in

254 hydrogenation than dehydrogenation reactions. The activity of KetoA hydrogenation

255 was 5 times higher than that of HYA dehydrogenation (Fig. 2).

256 Although many SDRs have high enantioselectivity [19-23], CLA-DH had low

257 enantioselectivity to dehydrogenate both $(R)$ and $(S)$ hydroxy fatty acids (Table1) and

258 produce $(R)$ and $(S)$ hydroxy fatty acids from oxo fatty acid. In addition, CLA-DH

259 dehydrogenated 10-, 12-, and 13-hydroxy fatty acids (Table 1), indicating its low 260 regioselectivity.

261 In our previous study, we reported the production of many kinds of hydroxy fatty 262 acids such as 10- and 13-hydroxy octadecapolyenoic acid [6,11,13,14]. Using these various hydroxy fatty acids and hydroxy fatty acid dehydrogenase such as CLA-DH, we can provide many kinds of corresponding oxo fatty acids by applying the wide substrate specificity of CLA-DH. These results enable us to provide new functional lipids, oxo fatty acids.

\section{Conclusions}


The properties of CLA-HY, a novel hydroxy fatty acid dehydrogenase from $L$.

271 hydroxy fatty acids with a preference to those with an internal hydroxy group. Such

272 substrate preference explained well that CLA-DH is involved in polyunsaturated fatty

273 acid saturation metabolism. From an application oriented perspective, CLA-DH is

274 useful for the production of oxo fatty acids with unique physiological functions in

275 combination with fatty acid hydratases such as CLA-HY $(11,13)$, which were reported

276 as good catalysts generating hydroxy fatty acids from common C18 fatty acids.

\section{ACKNOWLEDGMENTS}

This work was supported, in part, by the Industrial Technology Research Grant Program in 2007 (Grant 07A08005a to S.K.); the Project for the Development of a Technological

Infrastructure for Industrial Bioprocesses on Research and Development of New

Industrial Science and Technology Frontiers (S.S.) from the New Energy and Industrial

Technology Development Organization (NEDO) of Japan; Scientific Research Grants in

284 Aid 19780056 (to S.K.), 16688004 (to J.O.), and 18208009 (to S.S.); the Centers of 
287 Bio-Oriented Technology Research Advancement Institution of Japan (J.O.); Science 288 and Technology Promotion Program for Agriculture Forestry, Fisheries and Food 289 Industry from the Ministry of Agriculture, Forestry and Fisheries of Japan (J.O.); 290 Advanced Low Carbon Technology Research and Development Program of Japan 291 (S.K.); M.T. and S.K. received Research Fellowship from the Japan Society for the 292 Promotion of Science for Young Scientists.

\section{REFERENCES}

295 [1] Y. Park, K.J. Albright, W. Liu, J.M. Storkson, M.E. Cook, M.W. Pariza, Lipids. 32 $296 \quad$ (1997) 853-858.

297 [2] M.W. Pariza, Y.L. Ha, in: Y. Kuroda, D. Shankel, M.D. Waters (Eds.), 298 Antimutagenesis and Anticarcinogenesis Mechanism II, Plenum, New York, 1990, pp. $299 \quad 167-170$.

300 [3] C. Ip, S.F. Chin, J.A. Scimeca, M.W. Pariza, Cancer Res. 51 (1991) 6118-6124.

301 [4] K.N. Lee, D. Kritchevsky, M.W. Pariza, Atherosclerosis. 108 (1994) 19-25.

302 [5] Y.I. Kim, S. Hirai, T. Goto, C. Ohyane, H. Takahashi, T. Tsugane, C. Konishi, T. 303 Fujii, S. Inai, Y. Iijima, K. Aoki, D. Shibata, N. Takahashi, T. Kawada, PLoS One. 7 $304 \quad$ (2012) e31317. 
305 [6] S. Kishino, M. Takeuchi, S.B. Park, A. Hirata, N. Kitamura, J. Kunisawa, H. Kiyono,

306 R. Iwamoto, Y. Isobe, M. Arita, H. Arai, K. Ueda, J. Shima, S. Takahashi, K. Yokozeki,

307 S. Shimizu, J. Ogawa, Proc. Natl. Acad. Sci. USA. 110 (2013) 17808-17813.

308 [7] J. Ogawa, S. Kishino, A. Ando, S. Sugimoto, K. Mihara, S. Shimizu, J. Biosci.

309 Bioeng.100 (2005) 355-364.

310 [8] S. Kishino, J. Ogawa, Y. Omura, K. Matsumura, S. Shimizu, J. Am. Oil Chem. Soc. 31179 (2002) 159-163.

312 [9] S. Kishino, J. Ogawa, A. Ando, T. Iwashita, T. Fujita, H. Kawashima, S. Shimizu, 313 Biosci. Biotechnol. Biochem. 67 (2003) 179-182.

314 [10] S. Kishino, J. Ogawa, K. Yokozeki, S. Shimizu, Biosci. Biotechnol. Biochem. 75 315 (2011) 318-322.

316 [11] M. Takeuchi, S. Kishino, A. Hirata, S.B. Park, N. Kitamura, J. Ogawa, J. Biosci. 317 Bioeng. doi:10.1016/j.jbiosc.2014.10.022

318 [12] S. Kishino, S.B. Park, M. Takeuchi, K. Yokozeki, S. Shimizu, J. Ogawa, Biochem. 319 Biophys. Res. Commun. 416 (2011) 188-193.

320 [13] M. Takeuchi, S. Kishino, K. Tanabe, A. Hirata, S.B. Park, S. Shimizu, J. Ogawa, 321 Eur. J. Lipid Sci. Technol. 115 (2013) 386-393.

322 [14] J. Ogawa, K. Matsumura, S. Kishino, Y. Omura, S. Shimizu, Appl. Environ. 
323 Microbiol. 67 (2001) 1246-1252.

324 [15] R.G. Curtis, S.I. Heilbron, E.R.H. Jones, G.F. Woods, J. Chem. Soc. (1953)

$325 \quad 457-464$.

326 [16] E.G. Bligh, W.J. Dyer, Can. J. Biochem. Physiol. 37 (1959) 911-917.

327 [17] A.L. Gemal, J.L. Luche, J. Am. Chem. Soc. 103 (1981) 5454-5459.

328 [18] J.W. Song, E.Y. Jeon, D.H. Song, H.Y. Jang, U.T. Bornscheuer, D.K. Oh, J.B. Park, 329 Angew. Chem. 125 (2013) 2594-2597.

330 [19] N. Urano, S. Fukui, S. Kumashiro, T. Ishige, S. Kita, K. Sakamoto, M. Kataoka, S. 331 Shimizu, J. Biosci. Bioeng. 111 (2011) 266-271.

332 [20] N.H. Schlieben, K. Niefind, J. Muller, B. Riebel, W. Hummel, D. Schomburg, J. 333 Mol. Biol. 349 (2005) 801-813.

334 [21] A. Pennacchio, B. Pucci, F. Secundo, F.L. Cara, M. Rossi, C.A. Raia, Appl. 335 Environ. Microbiol. 74 (2008) 3949-3958.

336 [22] N. Itoh, K. Isotani, M. Nakamura, K. Inoue, Y. Isogai, Y. Makino, Appl. Microbiol. 337 Biotechnol. 93 (2012) 1075-1085.

338 [23] Y. Kallberg, U. Oppermann, H. Jornvall, B. Persson, Eur. J. Biochem. 269 (2002) $4409-4417$. 


\section{Fig. 1 SDS-PAGE analysis of purified CLA-DH}

343 Molecular mass standards: from the top, phosphorylase b $(97,200)$, bovine serum

344 albumin $(66,400)$, ovalbumin $(45,000)$, carbonic anhydrase $(29,000)$, and trypsin

345 inhibitor (20,100). The observed molecular weight of purified CLA-DH was $40 \mathrm{kDa}$.

346 Fig. 2 Effects of $\mathrm{NAD}^{+} / \mathrm{NADH}$ concentrations, temperature, and $\mathbf{p H}$ on the activity

347 of CLA-DH

348 (a) Effects of $\mathrm{NAD}^{+} / \mathrm{NADH}$ concentrations: Dehydrogenation activity (closed circles)

349 and hydrogenation activity (open circles) were assayed under standard reaction

350 conditions, except for $\mathrm{NAD}^{+} / \mathrm{NADH}$ concentrations. (b) Effects of temperature:

351 Dehydrogenation activity (closed circles) and hydrogenation activity (open circles) were assayed under standard reaction conditions, except for the temperature. (c) Effects of $\mathrm{pH}$ : Activity was assayed under standard reaction conditions, except for the buffers used.

Sodium citrate buffer (closed and open circles for dehydrogenation and hydrogenation, respectively), $\mathrm{pH}$ 3.0-4.0, and sodium succinate buffer (closed and open triangles for

356 dehydrogenation and hydrogenation, respectively), $\mathrm{pH}$ 4.0-5.5, were used.

(a) Effect of temperature: The thermal stability of the dehydrogenation activity (closed 
359 circles) and hydrogenation activity (open circles) were assessed under standard reaction conditions after incubation at each temperature $\left(18^{\circ} \mathrm{C}-67^{\circ} \mathrm{C}\right)$ for $30 \mathrm{~min}$. The activities

361 after incubation at $18^{\circ} \mathrm{C}$ were defined as $100 \%$ for dehydrogenation $(0.048 \mathrm{U} / \mathrm{mg})$ and hydrogenation (0.22 U/mg). (b) Effect of $\mathrm{pH}$ : The $\mathrm{pH}$ stabilities of the dehydrogenation

363 (closed) and hydrogenation (open) reactions were evaluated under standard reaction conditions after incubation at $37^{\circ} \mathrm{C}$ for $10 \mathrm{~min}$ at each $\mathrm{pH}$. Sodium citrate buffer, $\mathrm{pH}$ 3.0-4.0 (circles), sodium succinate buffer, $\mathrm{pH}$ 4.0-6.0 (triangles), potassium phosphate buffer, pH 5.0-7.5 (diamonds), and Tris-HCl buffer, 7.0-9.0 (squares) were used. The activities after incubation in sodium succinate buffer $(\mathrm{pH} 4.5)$ were defined as $100 \%$ for dehydrogenation (0.042 U/mg) and hydrogenation (0.22 U/mg).

Fig. 4 Multiple-sequence alignment of CLA-DH and ADHs belonging to the SDR family

The SDR family includes Rhodococcus erythropolis (AADH), Lactobacillus brevis (LbRADH), Thermus thermophilus (TtADH), and Leifsonia sp. strain S749 (LSADH). The accession numbers of the listed proteins are as follows: CLA-DH, BAL42247; AADH, BAF43657; LbRADH, YP_794544; TtADH, YP_003977; LSADH, BAD99642. 
376 TGXXXGXG is co-enzyme binding region in typical SDRs. The star indicates the four

377 members of catalytic tetrad.

378 


\title{
Marker
}

\author{
97,400 \\ 66,300 \\ 42,400
}

$\leftarrow$ CLA-DH

30,000

20,100

379

a

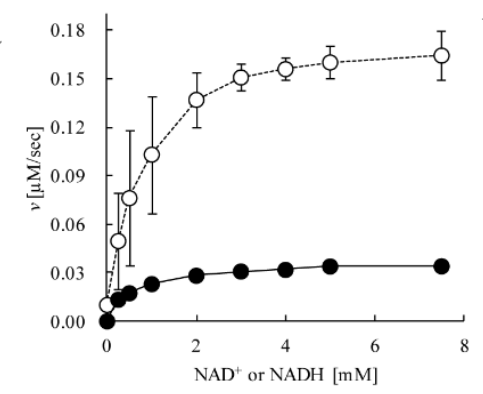

Fig. 1 SDS-PAGE analysis of purified CLA-DH
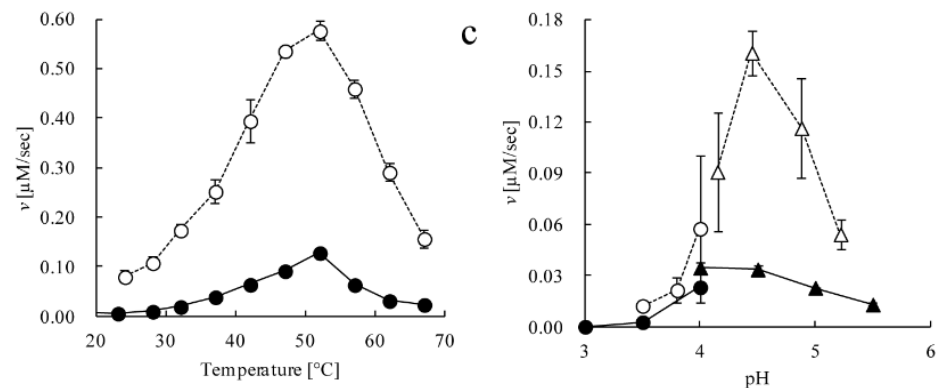

Fig. 2 Effects of $\mathrm{NAD}^{+} / \mathrm{NADH}$ concentrations, temperature, and $\mathrm{pH}$ on the activity

$$
\text { of CLA-DH }
$$


a

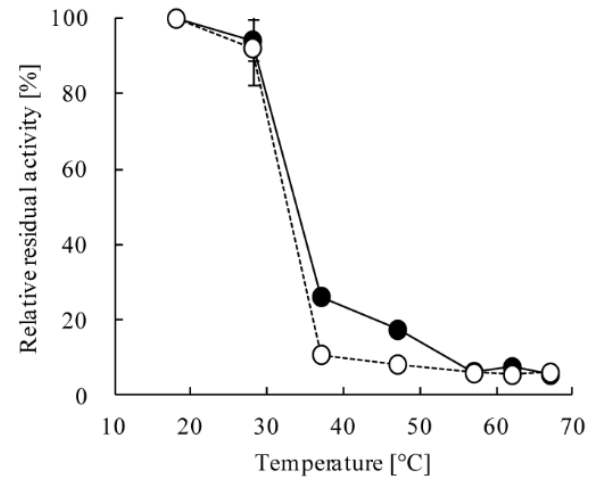

b

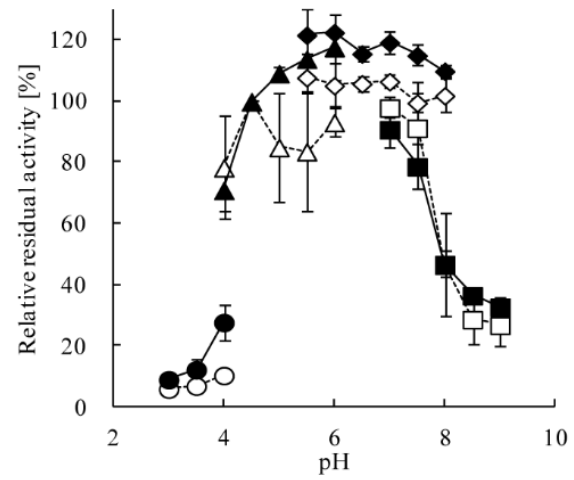

CLA-DH 1 --MKDFKDKVMFITGAAHGEGQVIAEGAADRGMKLTIVDIDEPALKKTYQHILDKGAEVL 58 AADH 1 -MFNSIEGRSVVVTGGSKGIGLGMVRVFARAGANVLMTARDALTLERAAEGLNGLPGAVS 59 LbRADH 1 -MSNRLDGKVAIVTGGTLGIGLAIATKFVEEGAKVMITGRHSDVGEKAAKSVGTPDQIQF 59 TtADH 1 MGLF--AGKGVLVTGGARGIGRAIAQAFAREGALVALCDLRPEGKE--VAEAIGGAFFQV 56 LSADH 1 MAQYDVADRSAIVTGGGSGIGRAVALTLAASGAAVLVTDLNEEHAQAVVAEIEAAGGKAA 60

CLA-DH 59 MVTADVTKEASVDDAVEQAMEKFGRIDLLINNAGIALP-GRIWELPTRDWEWIMHINLMS 117 AADH $\quad 60$ TLQVDVTNPDSLAGMAEVAAERHGGIDVLCANAGIFPS-KRLGEMTSEDMDSVFGVNVKG 118 LBRADH 60 FQHDSSDED-GWTKLFDATEKAFGPVSTLVNNAGIAVN-KSVEETTTAEWRKLLAVNLDG 117 TtADH 57 DLEDERERV-RFVEE---AAYALGRVDVLVNNAAIAAPGSAL-TVRLPEWRRVLEVNLTA 111 LSADH 61 ALAGDVTDP-AFGEASVAGANALAPLKIAVNNA IGGGEATVGDYSLDSWRTVIEVNLNA 119 CLA-DH 118 QVYAMKRVIPIMIQQKTHADILNVASIAGLVDT-PGMPSYHASKFASVGMTEATAYDLQR 176 AADH 119 TIHAVQACMPWLETSGRGRVVVTS-SITGPVTGYPGWSHYGASKAAQMGE IRTAAIELAP 177 LbRADH 118 VFFGTRLGIQRMKNKGLGASIINMSSIEGFV-GDPSLGAYNASKGAVRIMSKSAALDCAL 176 TtADH 112 PMHLSALAAREMRKVG-GGAIVNVASVQGLF-AEQENAAYNASKGGLVNLTRSLALDLAP 169 LSADH 120 VFYGMQPQLKAMAANG-GGAIVNMASIIGSV-GFANSSAYVTAKHALLGLTQNAALLYAA 177

CLA-DH 177 ANIDIDMHVMCPGFVQTDLYHTENHRPAQYSDPTDPYYQSEAYLKGQQFAKYVITNGKPI 236 AADH 178 KR--ITINAVLPGNVITEG---LDGLGQEY---LDQMASSVPAG----------SLGSVE 219 LbRADH 177 KDYDVRVNTVHPGYIKTP--LVDDLPGAE---EAMSQRTKTPMG-----------HIGEPN 221 TtADH 170 LR--IRVNAVAPGAIATEAVLEAIALSPDPERTRRDWEDLHALR----------RLGKPE 217 LSADH 178 DK--VRVVAVGPGFIRTP--LVEANLSAD---ALAFLEGKHALG----------RLGEPE 220

CLA-DH 237 DTIADTVFKALEDNRFYILTHPEYNPLIEDRVKRIVTDGAPDVHIMDGIM--- 286 AADH 220 DIANAALFFALDEAAYITG------------QSLIVDGGQVLPESAMALGEL 259 LBRADH 222 DIAYICVYLASNESKFATG------------SEFVVDGGYTAQ--------- 252 TtADH 218 EVAEAVLFLASEKASFITG------------AILPVDGGMTASFMMAGRPV- 256 LSADH 221 EVASLVAFLA ASDAASFITG-------------SYHLVDGGYTAQ--------- 251

392 Fig. 4 Multiple-sequence alignment of CLA-DH and ADHs belonging to the SDR 393 family 
Table 1 Substrate specificity of CLA-DH for dehydrogenation.

\begin{tabular}{|c|c|c|}
\hline 396 & Substrate & Relative activity [\%] \\
\hline 397 & (S)-10-Hydroxy-cis-12-octadecenoic acid (HYA) & $100^{\mathrm{a}}$ \\
\hline 398 & $(R)$-10-Hydroxy-cis-12-octadecenoic acid ((R)-HYA) & 98 \\
\hline 399 & 10-Hydroxyoctadecanoic acid & 25 \\
\hline 400 & 10-Hydroxy-trans-11-octadecenoic acid & 69 \\
\hline 401 & (S)-10-Hydroxy-cis-12,cis-15-octadecadienoic acid & 75 \\
\hline 402 & (S)-10-Hydroxy-cis-6,cis-12-octadecadienoic acid & 54 \\
\hline 403 & (R)-12-Hydroxy-cis-9-octadecenoic acid & 62 \\
\hline 404 & 13-Hydroxy-cis-9-octadecenoic acid & 142 \\
\hline 405 & 3-Hydroxyoctadecanoic acid (C18) & $-b$ \\
\hline 406 & 3-Hydroxytetradecanoic acid (C14) & - \\
\hline 407 & 2-Hydroxyeicosanoic acid (C20) & - \\
\hline 408 & Methyl (S)-10-Hydroxy-cis-12-octadecenoate & 127 \\
\hline 409 & 8-Hexadecanol & 24 \\
\hline
\end{tabular}

410 a, The activity of (S)-10-hydroxy-cis-12-octadecenoic acid dehydrogenation $(=0.048$

$411 \mathrm{U} / \mathrm{mg}$ ) under the condition (5 $\mathrm{mM} \mathrm{NAD}^{+}$; $37^{\circ} \mathrm{C}$, $\mathrm{pH} 4.5,15 \mathrm{~min}$ ) was defined as $100 \%$.

$412 \quad$ b-, not detected.

413 
414 Table 2 Substrate specificity of CLA-DH for hydrogenation.

\begin{tabular}{llc}
415 & Substrate & Relative activity [\%] \\
\cline { 2 - 3 } 416 & 10-Oxo-cis-12-octadecenoic acid (KetoA) & $100^{\mathrm{a}}$ \\
417 & 10-Oxooctadecanoic acid & 66 \\
418 & 10-Oxo-trans-11-octadecenoic acid & 53 \\
419 & 10-Oxo-cis-12,cis-15-octadecadienoic acid & 44 \\
420 & 10-Oxo-cis-6,cis-12-octadecadienoic acid & 67 \\
421 & 12-Oxo-cis-9-octadecenoic acid & 156 \\
422 & 13-Oxo-cis-9-octadecenoic acid & 170 \\
423 & Methyl 10-oxo-cis-12-octadecenoate & 332
\end{tabular}

425 a, The activity of 10-oxo-cis-12-octadecenoic acid hydrogenation $(=0.22 \mathrm{U} / \mathrm{mg})$ under 426 the condition (5 mM NADH; $37^{\circ} \mathrm{C}, \mathrm{pH} 4.5,15 \mathrm{~min}$ ) was defined as $100 \%$. 